\title{
Modafinil in Endozepine Stupor. A Case Report
}

\author{
Sharon Scott, Iftekhar Ahmed
}

\begin{abstract}
Background: Endozepine-4 stupor is a rare disorder. It presents with recurrent episodes of stupor and coma. Endozepine-4 levels are elevated during an attack with electroencephalogram showing fast activity during the ictal phase. There is no specific treatment and various drugs have been tried without long-term benefit. Method: We used the vigilance promoting drug modafinil to prevent such attacks in a case presented with endozepine stupor. Results: The vigilance promoting drug modafinil prevented recurrent episodes of stupor and coma in this patient at a dose of $200 \mathrm{mg} /$ day for a period of six months. Upon withdrawal of the drug, the patient experienced recurrent episodes and on reinstitution of modafinil she has remained asymptomatic in the following one year.
\end{abstract}

RÉSUMÉ: Le modafinil dans la stupeurà l'endozépine. Étude de cas. Introduction: La stupeur à l'endozépine4 est une maladie rare, qui se manifeste par des épisodes récurrents de stupeur et de coma. Les niveaux d'endozépine-4 sont élevés pendant les crises et l'électroencéphalogramme révèle une activité rapide pendant la phase ictale. Il n'existe pas de traitement spécifique de cette pathologie et plusieurs médicaments ont été essayés sans bénéfice à long terme. Méthode: Nous avons utilisé le modafinil, un médicament qui augmente la vigilance, pour prévenir les crises chez un cas de stupeur à l'endozépine. Résultats: Le modafinil, à la dose de 200 mg par jour, a prévenu les épisodes de stupeur et de coma chez cette patiente pendant six mois. Quand le médicament a été cessé, les crises sont revenues et ont cessé de nouveau à la reprise du médicament. La patiente demeure asymptomatique un an plus tard.

Can. J. Neurol. Sci. 2004; 31: 409-411

Endozepine stupor, a syndrome recognized in the past ten years, may be more common than previously thought. Several patients who have presented with episodes of recurrent stupor have been described previously. ${ }^{1-4}$ These patients present with elevated levels of an endogenous benzodiazepine-like substance, endozepine- 4 in the cerebrospinal fluid and serum when measured during stuporous episodes, ${ }^{1,3,4}$ and all of the patients exhibited a similar prodrome including fatigue and slurred speech, followed by stupor and coma. ${ }^{1-4}$ During the episodes, patients cannot be awakened. They showed decreased deep tendon reflexes and muscle tone, miotic, but reactive pupils and 13 to $20 \mathrm{~Hz}$ low amplitude activity on electroencephalogram (EEG) that normalized with the administration of the benzodiazepine receptor blocker flumazenil. ${ }^{1-5}$ Episodes of stupor last for hours to days and there is spontaneous recovery coinciding with the return of spinal fluid and serum endozepine levels to normal. ${ }^{1,2,4}$ Lugaresi et $\mathrm{al}^{1}$ studied twenty patients, all of whom exhibited the above symptoms and had 14 to $16 \mathrm{~Hz}$ beta activity on EEG that normalized with the administration of intravenous flumazenil. Extensive testing was performed on these individuals during and in between stuporous episodes, including toxicology screens, serum and urine chemistries, brain imaging, lumbar puncture and multiple sleep latency testing to rule out other causes of stupor. ${ }^{1}$ The fact that the endozepine levels returned to normal when the patients awakened and that the episodes were reversed with flumazenil have suggested that the episodes are caused by the effect of endozepine- 4 on the benzodiazepine receptor. ${ }^{1,2,5}$ Lugaresi et al $^{1}$ subsequently recommended the use of liquid chromatography mass spectrometry, the only objective way of documenting endozepine levels.

We describe a patient who has similar episodes of recurrent stupor as the patients described in previous reports. She was found to have an elevated endozepine-4 level in the serum during an episode. The medication modafinil (Provigil) was given in an attempt to prevent attacks of stupor and coma. Modafinil prevented these episodes for the next six months. On withdrawal of the drug, this patient experienced recurrent episodes of stupor and comatose state. Upon reinstituting modafinil, the patient had not suffered any further episodes in the next one year of follow-

From the Department of Family Practice, Trinity Lutheran Hospital, (SS); Department of Neurology, Research Medical Center, and Department of Neurology, University of Missouri, (IA); Kansas City, Missouri, USA.

ReCEIVED August 8, 2003. ACCEPTED INFINALFORM JANUARY 15, 2004. Reprint requests to: Iftekhar Ahmed, 6675 Holmes, Suite 425, Kansas City, Missouri 64131 USA. 
up. Modafinil may be a better drug in patients with endozepine stupor than those previously tried as its mechanism of action appears to involve the gamma-aminobutyric acid (GABA) system. It can also be given orally in once a day dosing and it has low abuse potential. ${ }^{6}$

\section{Case Report}

T.B. is a 77-year-old female who had a one to two year history of sudden episodes of loss of consciousness and stupor lasting for 24 to 48 hours. The patient had no other history of medical or cardiac problems. She was not on any medications. The episodes of stupor usually occurred in the morning, but occasionally occurred in the afternoon or late evening. The episodes would last anywhere between 8-12 hours, occasionally lasting up to 48 hours. During the attacks, the patient remained unresponsive. There was no history of any tonic-clonic activity. Patient has experienced a total of 6-8 episodes in the past year, a total of 14-15 episodes in the last $1 \frac{1}{2}$ years. The attacks were not associated with any postural changes and the patient had no control over any of the attacks. During the attacks, she would moan and groan and turn away from painful stimuli. She would resist attempt to eye opening. During recovery, the patient was confused, but gradually returned to her normal self. There was no family history of similar attacks, no history of environmental exposures related to the attacks and no history of illicit drug use.

The patient's blood pressure was 130/80, pulse 70, respirations 16 . General physical examination and breathing was normal. The patient's neurological examination showed she was stuporous, occulocephalic intact. Her pupils were $3 \mathrm{~mm}$, round and reactive. The patient would move upper and lower extremities in response to painful stimuli. The deep tendon reflexes were symmetric and plantar reflexes were flexor. No posturing could be elicited at any time. Her neck was supple and no bruits were noted.

The patient had multiple computerized axial topography head scans and magnetic resonance imaging done on various occasions and they were reported to be normal. Multiple drug screens, urine/serum, were negative including liquid chromatography mass spectrometry. An EEG done during the ictal phase showed generalized fast activity bilaterally with a frequency of around 14 to $16 \mathrm{~Hz}$. No paroxysmal activity was noted. No rapid eye movement sleep was seen. In the interictal phase the patient's EEG did not show any fast activity and was reported as normal. A spinal fluid examination was unremarkable, including bacterial and fungal antigens, cultures and polymerase chain reaction for viruses. Complete blood count was normal. Blood cultures were negative. Erythrocyte sedimentation rate was 10 . Chemistry profile including electrolytes, glucose and liver function tests were unremarkable. Chest $\mathrm{x}$-ray and electrocardiogram were normal. ANA and rheumatoid factor were negative. Serum protein electrophoresis was normal. Hormonal studies including T3, T4, TSH and cortisol were normal. A sleep study done during the interictal phase was also unremarkable. There was no evidence of sleep apnea or sleep onset rapid eye movement sleep and multiple sleep latency testing was unremarkable. The patient's serum endozepine- 4 level, measured during the ictal phase, was $450 \mathrm{pmol} / \mathrm{ml}$ which was markedly elevated (interictal level 0 ).

The levels were measured using chromatography-mass spectrometry as previously described. ${ }^{6}$

The patient was given a test dose of flumazenil 0.5 to $2 \mathrm{mg}$ on different occasions and showed improvement. The patient was subsequently given a trial of modafinil, $200 \mathrm{mg}$ a day, in an attempt to prevent attacks. After six months on modafinil, she had not had any attacks. Modafinil was subsequently withdrawn. In the following six months the patient started experiencing similar attacks again, and in the first two months after withdrawal of modafinil, three attacks were recorded. Modafinil was reinstituted and no further episodes recurred in the following year.

\section{Discussion}

In the past 11 years, a new neurologic syndrome has been described with recurrent stupor and coma. Tinupor et $\mathrm{al}^{4}$ referred to this syndrome of repeated episodes of spontaneous stupor and coma as idiopathic recurrent stupor and coma, also known as endozepine stupor. Endozepine stupor has not been previously reported in the United States. The syndrome was reported from multiple regions of Italy and it had been speculated that the etiology of the syndrome may be related to regional factors. However, patients with elevated endozepine levels during stuporous attacks have since been reported in Canada and South Africa. ${ }^{1,3}$ Our patient has exhibited the same clinical syndrome and, in fact, has also been found to have elevated endozepine levels in the blood during an episode of coma.

An EEG is helpful in diagnosis, typically showing faster activity. The EEG recordings in the majority of the previously reported patients revealed diffuse $13-20 \mathrm{~Hz}$ beta activity ${ }^{1-4}$ (one of the patients exhibited 10 to $11 \mathrm{~Hz}$ alpha activity) ${ }^{5}$ that was unresponsive to eye opening or pain.

This fast EEG activity is often seen in drug toxicity, particularly benzodiazepine intoxication, ${ }^{3}$ however drug screens done on all of these patients did not reveal any exogenous benzodiazepines. ${ }^{1,3-5}$ The administration of intravenous flumazenil in all patients transiently changed the EEG tracing to the $9 \mathrm{~Hz}$ activity associated with awakening and the patients did arouse briefly, ${ }^{1-5}$ but the effect of flumazenil in the intravenous form lasted only 10 to 15 minutes. ${ }^{1}$ The patients would then fall back into a stupor and the EEG activity would return to the 13 to $20 \mathrm{~Hz}$ fast activity. ${ }^{1,5}$ A trial of intravenous flumazenil at the onset of symptoms may further help to support the diagnosis while awaiting the results of endozepine levels. There does not appear to be an urgency to abort the acute attack as all of the patients spontaneously recovered within hours to days with no apparent residual effects. ${ }^{1-5}$

Treatment options for patients who experience endozepine stupor had been limited. The half-life of intravenous flumazenil is extremely short and its effects last only 10 to 15 minutes. The intravenous form is also limited in that it can only be given after an attack has begun. Ideally, a preventive medication is needed, but efforts so far have failed. Flumazenil had been given subcutaneously in an attempt to prevent attacks but because of poor absorption and a short half-life, results were not encouraging. ${ }^{5}$ Oral flumazenil is both difficult to obtain and has side effects including nausea and anxiety that limit its use. ${ }^{1}$ Lugaresi et al ${ }^{1}$ did attempt to prevent attacks in five patients with $20 \mathrm{mg} /$ day of oral flumazenil. The drug appeared to reduce the number of attacks in three of the patients, however in two patients, the trial was stopped secondary to side effects of the medication. Atropine and methylphenidate were used as well as subcutaneous flumazenil. Oral methylsergide, clonidine, piracetam and phenytoin all failed to prevent attacks. ${ }^{5}$ Modafinil 
has been successfully used as a vigilance enhancer. It has different neurochemical profile of action than amphetamines, suggesting that it doesn't involve a dopaminergic mechanism. ${ }^{6}$

The medial region of the hypothalamic large nuclei, including preoptic and posterior hypothalamic nuclei, function in behavioral states and arousal. They have projections to the association cortex including the anterior cingulate gyri. The posterior hypothalamus is thought to contribute to the production of wakefulness. ${ }^{7}$ Modafinil had been shown to reduce the extracellular concentration of GABA while increasing the extracellular levels of glutamate as measured by microdialysis techniques in the medial preoptic area and the posterior hypothalamus. ${ }^{7-9}$ It appears that slow wave sleep is associated with increased GABA levels in the medial preoptic nucleus and the posterior hypothalamus. ${ }^{7,8}$ Therefore, it has been speculated that reducing GABA levels in this area, along with the indirect increase in glutamate levels, might promote wakefulness. ${ }^{7}$ Endozepines bind presumably to the alpha subunit of $\mathrm{GABA}_{\mathrm{A}}$ receptor, as do the exogenous benzodiazepines, to act as allosteric activators of the receptor. Activation of the $\mathrm{GABA}_{\mathrm{A}}$ receptor causes opening of chloride channels thereby causing neuronal inhibition. The GABA binds to the beta subunit of the same receptor, directly opening the chloride channels. ${ }^{8}$

Modafinil appears to reduce the level of the neurotransmitter GABAin the medial preoptic area and posterior hypothalamus as described above. ${ }^{7,8}$ It may be that reducing the amount of circulating GABA counteracts the effect of endozepines or benzodiazepines because the benzodiazepines only act as modulators of the receptor and do not directly cause ion channel opening or neuronal inhibition. In other words, endozepines are unable to activate chloride channels without GABA binding. Suggested mechanisms of action of modafinil involve the enhancement of serotonin release with electrical stimulation of the nerve, in contrast to the spontaneous release of serotonin caused by fenfluoramine. ${ }^{10}$ Modafinil's affects appear to be blocked by pretreatment with a serotonergic antagonist. ${ }^{8,10}$ It has therefore been suggested that modafinil affects GABA and glutamate through a serotonergic mechanism. ${ }^{7,10,11}$

Recently, it has been shown that modafinil activates orexin containing neurons that may be involved in sleep/wake cycle regulation. ${ }^{12}$ Scamelli et al, ${ }^{12}$ in order to define modafinil's precise mechanism administered a range of doses of modafinil to rats, recorded sleep/wake activity and studied the pattern of neuronal activation using Fos-immunochemistry. Modafinil was administered at midnight during normal waking of rats. In addition to determining the influence of circadian rhythm, it was injected at noon on a normal light/dark cycle or in constant darkness. They found that at a dose of $75 \mathrm{mg} / \mathrm{kg}$ of modafinil increased Fos immunoreactivity in the tuberomaxillary nucleus and in orexin (hypocretin) neurons in the perifornical area, two cell groups implicated in the regulation of wakefulness. More research is needed to look at the relationship between orexins and stupor and coma.

Modafinil, in our patient, appears to have reduced the frequency of attacks of endozepine stupor. Spontaneous remission in endozepine stupor does occur, but our patient relapsed after discontinuing the drug and with reinstitution the patient improved, indicating modafinil is helpful in controlling these attacks. Further trials are needed to study modafinil as treatment for these patients, however a randomized controlled trial would be difficult due to the small numbers of patients with this disorder. Modafinil may be a more effective treatment than those previously tried. It would be a good choice as a preventive medication because of its once-a-day oral dosing, low incidence of side effects and its low abuse potential.

\section{REFERENCES}

1. Lugaresi E, Montagna P, Tinupor P. Endozepine stupor: recurring stupor linked to endozepine-4 accumulation. Brain 1998;121: 127-133.

2. Rothstein JD, Guido Ai A, Tinupor P, et al. Endogenous benzodiazepine receptor ligands in idiopathic recurring stupor. Lancet 1992; 340: 1002-1004.

3. Chen R, Rothstein JD, Frey KA. Idiopathic recurring stupor: a new syndrome. Neurology 1995;45(Suppl 4):A177.

4. Tinupor P, Montagna P, Cortelli P. Idiopathic recurring stupor: a case with possible involvement of the gamma-aminobutyric acid (GABA)ergic system. Ann Neurol 1992; 31(5):503-506.

5. Lotz BP, Schutte CM, Bartel PR. Recurrent attacks of unconsciousness with diffuse EEG alpha activity. Sleep 1993; 16(7):671-677.

6. Rothstein JD, Garland W, Pvia G, et al. Purification and characterization of naturally occurring benzodiazepine receptor ligands in rat and human brain. J Neurochem 1992; 6(58):21022115.

7. Jasinski D, Ruzica M, Listanovic K. Evaluation of the abuse liability of modafinil and other drugs for excessive daytime sleepiness associated with narcolepsy. Clin Neuropharmacol 2000; 23(3): 149-156

8. Ferraro L, Tanganelli S, O'Connor WT. The vigilance promoting drug modafinil increases extracellular glutamate levels in the medial preoptic area and the posterior hyothalamus of the conscious rat: prevention by local $\mathrm{GABA}_{\mathrm{A}}$ receptor blockade. Neuropsychopharmacology 1999; 20(4): 346-356.

9. US Modafinil in Narcolepsy Multicenter Study Group. Randomized trial of modafinil as a treatment for the excessive daytime somnolence of narcolepsy. Neurology 2000; (54): 1166-1175.

10. Ferraro L, Fuxe K, Tanganelle S, et al. Amplification of cortical seratonin release: a further neurochemical action of the vigilancepromoting drug modafinil. Neuropharmacology 2000; 39(11): 1974-1983.

11. Azuma S, Kodernia T, Honda K. State dependent changes of extracellular glutamate in the medial area in freely behaving rats. Neurosci Letts 1996; 214: 179-182.

12. Scamelli TE, Eastabrook IV, McCarthy MT. Hypothalamic arousal regions are activated during modafinil-induced wakefulness. J Neurosci 2000; 20(22): 8620-8628. 